S. Kondo

Nagoya Math. J.

Vol. 99 (1985), 11-30

\title{
TYPE II DEGENERATIONS OF K3 SURFACES
}

\author{
SHIGEYUKI KONDO
}

\section{Introduction}

A degeneration of $K 3$ surfaces (over the complex number field) is a proper holomorphic map $\pi: X \rightarrow \Delta$ from a three dimensional complex manifold to a disc, such that, for $t \neq 0$, the fibres $X_{t}=\pi^{-1}(t)$ are smooth $K 3$ surfaces (i.e. surfaces $X_{t}$ with trivial canonical class $K_{X_{t}}=0$ and $\operatorname{dim} H^{1}\left(X_{t}, \mathcal{O}_{X_{t}}\right)$ $=0)$.

Recently, Kulikov [7], Persson and Pinkham [12] have classified the semi-stable degenerations of $K 3$ surfaces into three types and Friedman [2], [3] has studied the local moduli problem for $D$-semi-stable $K 3$ surfaces. On the other hand, Piatetskii-Shapiro and Shafarevich [13], Burns and Rapoport [1] proved the Torelli theorem for Kaehler $K 3$ surfaces. One of the next steps for the study of the moduli problem for $K 3$ surfaces is to extend the theory of the period of smooth $K 3$ surfaces to the degenerate case.

From the point of view of the moduli problem, the following surfaces are fundamental (see (1.6)): A stable $K 3$ surface of type II is a surface $X=X_{1} \cup X_{2}$ with normal crossings such that; (i) $X_{i}$ is a smooth rational surface $(i=1,2)$ and $E=X_{1} \cap X_{2}$ is a smooth elliptic curve, (ii) the dualizing sheaf $\omega_{X}$ on $X$ is trivial, (iii) the line bundle $N_{E / X_{1}} \otimes N_{E / X_{2}}$ over $E$ is trivial, where $N_{E / X_{i}}$ is the normal bundle of $E$ in $X_{i}(i=1,2)$.

In this paper we define the periods of stable $K 3$ surfaces of type II and prove the Torelli theorem for them. Let $X=X_{1} \cup X_{2}$ be a stable $K 3$ surface of type II. Then the component $X_{i}$ is not always minimal and there happens a birational modification between the stable $K 3$ surfaces of type II, which is called a modification of type $I$ in [7]. Let $L(X)$ denote the lattice $\left\{\left(x_{1}, x_{2}\right) \in H^{2}\left(X_{1} ; Z\right) \oplus H^{2}\left(X_{2} ; Z\right) ;\left(x_{1},\left[E_{1}\right]\right)_{X_{1}}=\left(x_{2},\left[E_{2}\right]\right)_{X_{2}}\right\} / Z\left(\left[E_{1}\right]-\right.$ $\left.\left[E_{2}\right]\right)$, where $\left[E_{i}\right]\left(\in H^{2}\left(X_{i} ; Z\right)\right)$ is the cohomology class of the double curve $E=X_{1} \cap X_{2}$. Then $L(X)$ is an even unimodular lattice of signature $(1,17)$.

Received October 27, 1983. 
We define the period of the stable $K 3$ surface $X$ by a homomorphism $\omega_{X}: L(X) \rightarrow J(E):=$ Jacobian variety of $E$ (see (2.8)). The idea of our definition is due to Y. Namikawa [11].

Roughly speaking, our main result is as follows: let $X$ and $X^{\prime}$ be two stable $K 3$ surfaces of type II with the "same" period. Then there is a bimeromorphic map $X \longrightarrow X^{\prime}$ which is a composite of modifications of type I (see (2.14)).

The plan of this paper is as follows: in Section 1 we collect the known facts about the semi-stable degenerations of $K 3$ surfaces, in Section 2 we state our main results (Theorems (2.14), (2.15)), and Section 3 is devoted to their proofs.

I would like to express my thanks to Professor Yukihiko Namikawa whose insight and encouragement are invaluable.

\section{§1. Semi-stable degenerations of $K 3$ surfaces}

(1.1) A semi-stable degeneration of surfaces (resp. K3 surfaces) is a proper holomorphic map $\pi: X \rightarrow \Delta$ from a three dimensional complex manifold to a disc such that: (i) the fibres $X_{t}=\pi^{-1}(t)$ are smooth surfaces (resp. smooth $K 3$ surfaces) for $t \neq 0$; (ii) the central fibre $X_{0}=\pi^{-1}(0)$ is a divisor with normal crossings; (iii) all components of $X_{0}$ have multiplicity one in the fibre.

If a degeneration of surfaces is projective, it becomes bimeromorphic to a semi-stable one after a base change ([5]).

(1.2) Let $\pi: X \rightarrow \Delta$ be a semi-stable degeneration of surfaces. The dual graph of $X_{0}=\pi^{-1}(0)$ is the following simplicial complex: (i) The set of vertices is the set of irreducible components of $X_{0}$; (ii) The set of edges is the set of components of double curves of $X_{0}$; (iii) The set of faces is the set of triple points of $X_{0}$.

(1.3) A degeneration of surfaces $\pi: X \rightarrow \Delta$ is weakly Kaehler if there exists a bimeromorphic map $\phi: X \rightarrow X^{\prime}$ such that $\phi$ is biholomorphic on $X-\pi^{-1}(0)$, the diagram

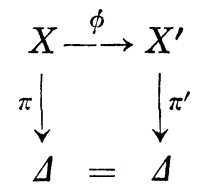

is commutative and such that $X^{\prime}$ is a Kaehler manifold. 
In the study of the degenerations of $K 3$ surfaces, the following results. are essential.

(1.4) Theorem (Kulikov [7], Persson and Pinkham [12]). Let $\pi: X \rightarrow \Delta$ be a degeneration of $K 3$ surfaces. If all components of the central fibre $X_{0}=\pi^{-1}(0)$ are algebraic, then $X$ is bimeromorphic to a semi-stable degeneration $\pi^{\prime}: X^{\prime} \rightarrow \Delta$ with $K_{X^{\prime}} \equiv \mathcal{O}_{X^{\prime}}$, where $K_{X^{\prime}}$ is the canonical line bundle of $X^{\prime}$.

(1.5) Theorem (Kulikov [7]). Let $\pi: X \rightarrow \Delta$ be a weakly Kaehler, semistable degeneration of $K 3$ surfaces with $K_{X} \equiv \mathcal{O}_{X}$. Then $X_{0}=\pi^{-1}(0)$ is one of the following three types:

(Type I) $X_{0}$ is a smooth K3 surface;

(Type II) $X_{0}=V_{1}+V_{2}+\cdots+V_{n-1}+V_{n}$, where $V_{1}$ and $V_{n}$ are rational surfaces, $V_{2}, \cdots, V_{n-1}$ are elliptic ruled surfaces and $V_{i} \cap V_{i+1}, i=1, \cdots, n-1$, are smooth elliptic curves. The dual graph of $X_{0}$ is as follows:

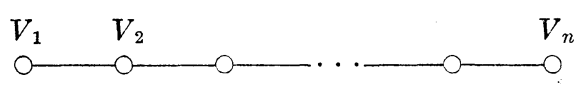

(Type III) $X_{0}=V_{1}+\cdots+V_{n}$, where all $V_{i}$ 's are rational surfaces and the double curves $V_{i} \cap V_{j}$ on $V_{j}$ are smooth rational curves forming a cycle. The dual graph of $X_{0}$ is a triangulation of 2-sphere $S^{2}$.

(1.6) Remark. In this paper we study the type II degenerations in the above Theorem (1.5). Among them, the type II degenerations without the elliptic ruled components are fundamental in the following sense: let $\pi: X \rightarrow \Delta$ be as in Theorem (1.5). Suppose the central fibre $X_{0}=V_{1}+V_{2}$ $+\cdots+V_{n-1}+V_{n}$ is of type II. By performing some birational modifications, we can assume that the elliptic ruled components $V_{2}, \cdots, V_{n-1}$ are minimal. Then we can contract $V_{2}, \cdots, V_{n-1}$ along the rulings for which the double curves are sections (cf. [2], [4]). This produces a new threefold $X^{\prime}$ mapping to $\Delta$, and $X^{\prime}$ has a curve of $A_{n-2}$ surface singularities. Moreover the new central fibre $X_{0}^{\prime}$ is a surface of typa II without the elliptic ruled components. This is similar to the case of degenerations of elliptic curves of type $I_{b}$ ([6], p. 604).

(1.7) Lemma. Let $\pi: X \rightarrow \Delta$ be as in Theorem (1.5). Suppose $X_{0}$ is of type II and without the elliptic ruled component: i.e. $X_{0}=X_{1} \cup X_{2}$, where 
$X_{i}$ is a rational surface $(i=1,2)$ and $E=X_{1} \cap X_{2}$ is a smooth elliptic curve. Then

(i) $E \in\left|-K_{X_{i}}\right|(i=1,2)$;

(ii) $N_{E / X_{1}} \otimes N_{E / X_{2}} \cong \mathcal{O}_{E}$;

(iii) $\left(E^{2}\right)_{X_{1}}+\left(E^{2}\right)_{X_{2}}=0$.

Proof. By the adjunction formula and $\mathcal{O}_{X}\left(X_{0}\right)=\mathcal{O}_{X}$, we have $K_{X_{1}}=$ $\left.\left[K_{X}+X_{1}\right]\right|_{X_{1}}=\left.\left[X_{1}\right]\right|_{X_{1}}=-\left.\left[X_{2}\right]\right|_{X_{1}}$. Hence $E \in\left|-K_{X_{1}}\right|$. Since $N_{E / X_{1}}=\left.\mathcal{O}_{X}\left(X_{2}\right)\right|_{E}$ and $N_{E / X_{2}}=\left.\mathcal{O}_{X}\left(X_{1}\right)\right|_{E}$,

$$
N_{E^{\prime} / X_{1}} \otimes N_{E / X_{2}}=\left.\left.\mathcal{O}_{X}\left(X_{1}\right)\right|_{E} \otimes \mathcal{O}_{X}\left(X_{2}\right)\right|_{E}=\left.\mathcal{O}_{X}\left(X_{0}\right)\right|_{E}=\mathcal{O}_{E}
$$

Now the statement (iii) is obvious.

(1.8) Definition. A stable K3 surface of type II (resp. a quasi-stable $K 3$ surface of type II) is a surface $X=X_{1} \cup X_{2}$ with normal crossings such that $X_{i}$ is a smooth rational surface, $E=X_{1} \cap X_{2}$ is a smooth elliptic curve and satisfies the following conditions: (i) $E \in\left|-K_{X_{i}}\right|(i=1,2)$; (ii) $N_{E / X_{1}} \otimes$ $N_{E / X_{2}} \cong \mathcal{O}_{E}$ (resp. (i) $E \in\left|-K_{x_{i}}\right|(i=1,2)$; (ii') degree $\left(N_{E / X_{1}} \otimes N_{E / X_{2}}\right)=0$ ).

(1.9) Remark. More generally, Friedman ([2], [3]) has defined the $D$ semi-stable $K 3$ surfaces (cf. [3], (5.5)). We remark here that a quasi-stable $K 3$ surface of type II is $D$-semi-stable if and only if it satisfies the condition (ii) in (1.8) (i.e. stable).

Every stable $K 3$ surface of type II is obviously quasi-stable. In Sections 2 and 3, we shall treat a quasi-stable $K 3$ surface of type II rather than a stable one. The following result states that every stable $K 3$ surface of type II is nothing but a degenerate fibre of a semi-stable degeneration of $K 3$ surfaces.

(1.10) Theorem (Friedman [2], [3]). Let $X$ be a stable K3 surface of type II. Then the Kuranishi space of $X$ looks like $V_{1} \cup V_{2}$, here

(1) $V_{1}, V_{2}$ are smooth and meet transversally;

(2) $\operatorname{dim} V_{1}=\operatorname{dim} H^{1}\left(X, \theta_{X}\right)=20, \operatorname{dim} V_{2}=20$ and $\operatorname{dim}\left(V_{1} \cap V_{2}\right)=19$, where $\theta_{X}$ is a sheaf of derivations of $\mathcal{O}_{X}$;

(3) $V_{1}$ is a space corresponding to the topologically trivial deformations;

(4) Let $X_{t}$ be a surface corresponding to a point $t \in V_{1} \cup V_{2}$. Then

(i) $X_{t}$ is a smooth $K 3$ surface if $t \in V_{2}-V_{1}$.

(ii) $X_{t}$ is a quasi-stable $K 3$ surface of type II if $t \in V_{1}$,

(iii) $X_{t}$ is a stable $K 3$ surface of type II if and only if $t \in V_{1} \cap V_{2}$. 
(1.11) Remark. In [2], [3], Friedman has showed the similar results for every $D$-semi-stable $K 3$ surface.

(1.12) A modification of type $\mathrm{I}$ is a birational modification of a stable $K 3$ surface as follows: Let $X=X_{1} \cup X_{2}$ be a stable $K 3$ surface of type II, $E=X_{1} \cap X_{2}$ the double curve and $C$ an exceptional curve of the first kind on $X_{1}$. Note that $C$ intersects at exactly one point with $E$ (see (1.13)). By (1.10) we regard $X$ as a cental fibre of a semi-stable degeneration of $K 3$ surfaces. Then $C$ can be moved to the adjacent component $X_{2}$;

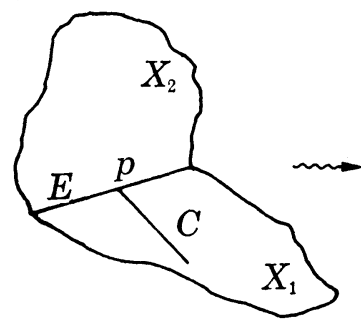

blow up $C$

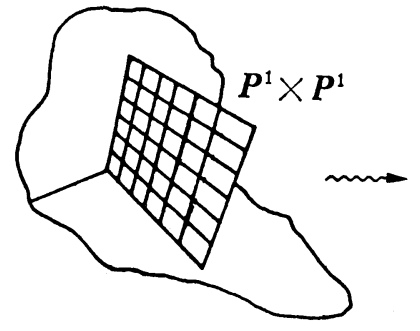

blow bown $\boldsymbol{P}^{1} \times \boldsymbol{P}^{1}$ in the other direction

For quasi-stable $K 3$ surfaces, the modification of type $I$ is defined as follows: on $X_{1}$, contracting $C$ to a point, and on $X_{2}$, blowing up at $p=$ $E \cap C$.

We close this section with two lemmas for quasi-stable $K 3$ surfaces.

(1.13) Lemma. Let $S$ be a component of a quasi-stable $K 3$ surface of type II and $C$ an irreducible curve on $S$ with $E \neq C$ and $\left(C^{2}\right)_{S}<0$. Then $C$ is a smooth rational curve such that either

(1) $\left(C^{2}\right)_{s}=-1,(C, E)_{s}=1$, or

(2) $\left(C^{2}\right)_{S}=-2,(C, E)_{s}=0$,

where $E \in\left|-K_{S}\right|$ is the double curve.

Proof. By $E \in\left|-K_{s}\right|$, the arithmetic genus of $C$ can be computed as follows: $2 p_{a}(C)-2=\left(C^{2}\right)_{S}-(C, E)_{S}$. The lemma (1.13) can be easily deduced from this formula.

(1.14) Lemma. Let $X=X_{1} \cup X_{2}$ be a quasi-stable $K 3$ surface of type II. Then the possible types for the relatively minimal model of $X_{i}$ are as follows: (a) $\boldsymbol{P}^{2}$, or (b) $\boldsymbol{F}_{n}, n=0,2$.

Proof. Let $E$ be the double curve of $X$. Let $\bar{X}_{i}$ be a relatively minimal model of $X_{i}(i=1,2)$. By the classification of surfaces, $\bar{X}_{i}$ is either $\boldsymbol{P}^{2}$ or $\boldsymbol{F}_{n}, n \geqq 0, n \neq 1$. We note that $\bar{X}_{i}$ has an anti-canonical divisor 
which is a smooth elliptic curve. If $\bar{X}_{i} \cong F_{n}$, then $-K_{F_{n}}=2 s_{n}+(n+2) R$, where $R$ is a fibre and $s_{n}$ is the section with $\left(s_{n}^{2}\right)=-n$. By the above remark, we have

$$
0 \leqq\left(-K_{F_{n}}, s_{n}\right)=-2 n+n+2=2-n, \text { and } n \leqq 2 .
$$

Hence we have proved (1.14).

(1.15) Remark. In the following sections, we assume that the selfintersection number $\left(E^{2}\right)_{X_{i}}$ is equal to zero, $i=1,2$ (see (2.4)). In this case, by (1.14), we can choose $P^{2}$ as a relatively minimal model of $X_{i}$ $(i=1,2)$.

\section{§2. Periods of stable $K 3$ surfaces and Torelli theorem}

In this section, we define the period of (quasi-) stable $K 3$ surfaces and we state the Torelli theorem. Our statement may be regarded as a degenerate case of the Torelli theorem for Kaehler $K 3$ surfaces ([1]). In the following, we shall deal with quasi-stable $K 3$ surfaces of type II. For stable $K 3$ surfaces of type II, theorems (2.14), (2.15) are also true with some modifications of the period domain (see Remark (2.9), (ii)). For simplicity, we say a quasi-stable $K 3$ surface for a quasi-stable $K 3$ surface of type II.

(2.1) Let $X=X_{1} \cup X_{2}$ be a quasi-stable $K 3$ surface with the double curve $E$. The Mayer-Vietoris cohomology exact sequence is as follows:

$$
\begin{aligned}
0 \longrightarrow H^{1}(E ; Z) \longrightarrow H^{2}(X ; Z) \longrightarrow H^{2}\left(X_{1} ; Z\right) \oplus H^{2}\left(X_{2} ; Z\right) & \\
& \longrightarrow H^{2}(E ; Z) \longrightarrow 0 .
\end{aligned}
$$

Put ${ }^{0} W_{2}(X):=H^{2}(X ; Z),{ }^{0} W_{1}(X):=H^{1}(E ; Z)$, and we let ${ }^{0} L(X)$ denote the quotient module ${ }^{0} W_{2}(X) /{ }^{0} W_{1}(X)$. Then

$$
{ }^{0} L(X) \cong \operatorname{Ker}\left\{H^{2}\left(X_{1} ; Z\right) \oplus H^{2}\left(X_{2} ; Z\right) \longrightarrow H^{2}(E ; Z)\right\} .
$$

Under this isomorphism, we always regard an element of ${ }^{\circ} L(X)$ as a class in $H^{2}\left(X_{1} ; Z\right) \oplus H^{2}\left(X_{2} ; Z\right)$. Let $D_{i}$ be a divisor on $X_{i}$ and denote by $\left[D_{i}\right]$ the cohomology class of $D_{i}$. If an element $\left(\alpha_{1}, \alpha_{2}\right) \in{ }^{0} L(X)$ such that $\alpha_{i}$ is represented by $\left[D_{i}\right]$, we often denote $\left(\alpha_{1}, \alpha_{2}\right)$ by $\left[D_{1}\right]+\left[D_{2}\right]$. Let $E_{i}$ be the double curve on $X_{i}(i=1,2)$, then $\left[E_{1}\right]-\left[E_{2}\right]$ is contained in ${ }^{0} L(X)$ for $\left(E_{1}^{2}\right)_{X_{1}}+\left(E_{2}^{2}\right)_{X_{2}}=0$.

A lattice $H$ is a free abelian group of finite rank endowed with a 
integral quadratic form. The group $H^{2}\left(X_{1} ; Z\right) \oplus H^{2}\left(X_{2} ; Z\right)$ admits a canonical structure of a lattice induced from the cup product. Note that ${ }^{0} L(X)$ inherits a lattice structure from that of $H^{2}\left(X_{1} ; Z\right) \oplus H^{2}\left(X_{2} ; Z\right)$. We denote its bilinear form by $\langle$,$\rangle .$

(2.2) Remark. ${ }^{0} W_{2}(X),{ }^{0} W_{1}(X)$ are the weight filtrations of the mixed Hodge structure on $X$ ([7], p. 960).

In our study, the problem is how to interpret the modifications of type $I$ in the language of cohomology groups. The following lemma will be needed.

(2.3) Lemma ([2]). Let $X=X_{1} \cup X_{2}$ be a quasi-stable $K 3$ surface with the double curve $E$ and let $C$ be an exceptional curve of the first kind on $X_{1}$. Let $X^{\prime}=X_{1}^{\prime} \cup X_{2}^{\prime}$ be the quasi-stable $K 3$ surface with the double curve $E^{\prime}$ obtained by the modification of type I along $C$. We denote this modification by $\phi_{C}$. Then

$$
N_{E / / X_{1}^{\prime}} \otimes N_{E^{\prime} / X_{2}^{\prime}} \cong N_{E / X_{1}} \otimes N_{E / X_{2}}
$$

and $\phi_{C}$ induces a lattice isometry

$$
\phi_{C}^{*}:{ }^{0} L\left(X^{\prime}\right) \longrightarrow{ }^{0} L(X) .
$$

Proof. The first statement follows easily from definition. Lat $C^{\prime}$ ba the exceptional curve on $X_{2}^{\prime}$ created by $\phi_{C}$. Denote by

$$
\pi_{1}: X_{1} \longrightarrow X_{1}^{\prime} \quad \text { (resp. } \pi_{2}: X_{2}^{\prime} \longrightarrow X_{2} \text { ) }
$$

the blowing up at $p^{\prime}=E^{\prime} \cap C^{\prime}$ (resp. $\left.p=E \cap C\right)$. For $\left(\left[D_{1}^{\prime}\right],\left[D_{2}^{\prime}\right]\right) \in{ }^{0} L\left(X^{\prime}\right)$ such that $\left(D_{2}^{\prime}, C^{\prime}\right)_{X^{\prime}}=r$, we define $\phi_{C}^{*}$ by

$$
\phi_{C}^{*}\left(\left(\left[D_{1}^{\prime}\right],\left[D_{2}^{\prime}\right]\right)\right)=\left(\pi_{1}^{*}\left(\left[D_{1}^{\prime}\right]\right)+[r C],\left[\left(\pi_{2}\right)_{*} D_{2}^{\prime}\right]\right) .
$$

Then we can easily check that $\phi_{C}^{*}\left(\left[D_{1}^{\prime}\right]+\left[D_{2}^{\prime}\right]\right)$ is contained in ${ }^{\circ} L(X)$ and $\phi_{C}^{*}$ is isometric. We leave the proof to the reader.

(2.4) Assumption. From now on, we assume that the self-intersection number $\left(E_{i}^{2}\right)_{X_{i}}$ is equal to zero $(i=1,2)$. Since $\left(E_{1}^{2}\right)_{X_{1}}+\left(E_{2}^{2}\right)_{X_{2}}=0$, every quasi-stable $K 3$ surface satisfies this assumption, after performing some modifications of type $I$.

(2.5) Definition. We keep the notation of (2.1). Let $\pi_{i}: X_{i} \rightarrow \bar{X}_{i}$ be a relatively minimal model $(i=1,2)$. Here we choose $\bar{X}_{i} \cong \boldsymbol{P}^{2}$ (see (1.15)). By the assumption (2.4), $\pi_{i}: X_{i} \rightarrow P^{2}$ is the blowing up of $\boldsymbol{P}^{2}$ at nine points 
on a smooth elliptic curve. We denote the distinct exceptional curves of $\pi_{i}$ (not necessarily irreducible) which meet $E_{i}$ by $L_{i}^{1}, \cdots, L_{i}^{9}$. We suppose that they are indexed in such a way that $L_{i}^{k} \subset L_{i}^{k^{\prime}}$ implies that $k \geqq k^{\prime}$. Let $H_{i}$ be the total transform of the line in $\bar{X}_{i}=\boldsymbol{P}^{2}$ which passes through $\pi_{i}\left(L_{i}^{1}\right)$ and $\pi_{i}\left(L_{i}^{2}\right)$ (at least when $\pi_{i}\left(L_{i}^{1}\right) \neq \pi_{i}\left(L_{i}^{2}\right)$; otherwise take the tangent line of $\pi_{i}\left(E_{i}\right)$ at $\left.\pi_{i}\left(L_{i}^{1}\right)=\pi_{i}\left(L_{i}^{2}\right)\right)$. Note that the set $\left\{\left[H_{i}\right],\left[L_{i}^{1}\right], \cdots,\left[L_{i}^{9}\right]\right\}$ is a basis of $H^{2}\left(X_{i} ; Z\right)(i=1,2)$. Any indexed set of exceptional curves $\left\{L_{i}^{k}\right\}$ thus obtained will be called an exceptional-configuration of $X$. As $L_{i}^{k}$ is the unique effective divisor within its cohomology class $\left[L_{i}^{k}\right]$, we use the same terminology for the corresponding collection $\left\{\left[L_{i}^{k}\right]\right\}$.

(2.6) A basis of ${ }^{0} L(X)$ is given by $\left\{\left[E_{1}\right],\left[E_{2}\right],\left[L_{1}^{9}\right]+\left[L_{2}^{9}\right],\left[L_{i}^{k}\right]-\left[L_{i}^{k+1}\right]\right.$, $\left.\left[H_{i}\right]-\left[L_{i}^{1}\right]-\left[L_{i}^{2}\right]-\left[L_{i}^{3}\right] ; i=1,2, k=1, \cdots, 7\right\}$. We note that for all quasistable $K 3$ surfaces, their corresponding lattices ${ }^{0} L(X)$ are isometric each other. Let $L$ (resp. $F$ ) be an abstract lattice which is isometric to ${ }^{\circ} L(X)$ (resp. ${ }^{0} W_{1}(X)$ ) for some reference quasi-stable $K 3$ surface $X$ with the double curve $E$ and let $\theta$ be a vector in $L$ corresponding to $\left[E_{1}\right]-\left[E_{2}\right] \in{ }^{0} L(X)$.

(2.7) Definition. A marking of a quasi-stable $K 3$ surface $X$ with the double curve $E$ is a lattice isometry

$$
\alpha_{X}:{ }^{0} L(X) \oplus{ }^{0} W_{1}(X) \longrightarrow L \oplus F
$$

such that $\alpha_{X}\left({ }^{0} W_{1}(X)\right)=F$ and $\alpha_{X}\left(\left[E_{1}\right]-\left[E_{2}\right]\right)= \pm \theta$. We call the pair $\left(X, \alpha_{X}\right)$ a marked quasi-stable $K 3$ surface of type II.

Now we define the periods of quasi-stable $K 3$ surfaces. The idea of our definition is due to Y. Namikawa ([11]).

(2.8) Let $X$ be a quasi-stable $K 3$ surface with the double curve $E$. Let $\underline{\omega}_{X}$ be the dualizing sheaf of $X$ (i.e. let $f: \bar{X}=X_{1} \Perp X_{2} \rightarrow X$ be the normalization of $X$, with $E_{i}$ being the smooth elliptic curve on $X_{i}$ such that $f\left(E_{i}\right)=E(i=1,2)$. Then $\underline{\omega}_{X}$ is the sheaf of 2 -forms $\omega$ on $\bar{X}$ holomorphic except for simple poles at $E_{i}(i=1,2)$ and with $\operatorname{Res}_{E_{1}} \omega+\operatorname{Res}_{E_{2}} \omega$ $=0$ ). By definition (1.8), there is a nowhere vanishing section $\omega_{X}$ of $H^{0}\left(X, \underline{\omega}_{X}\right)$. Consider the exact homology sequence of the pair $\left(X_{i}, X_{i}-E\right)$ : $\cdots \rightarrow H_{3}(X ; Z) \rightarrow H_{3}\left(X_{i}, X_{i}-E ; Z\right) \stackrel{\partial}{\longrightarrow} H_{2}\left(X_{i}-E ; Z\right) \rightarrow H_{2}\left(X_{i} ; Z\right) \rightarrow H_{2}\left(X_{i}\right.$, $\left.X_{i}-E ; Z\right) \rightarrow \cdots$. We identify $H_{k}\left(X_{i}, X_{i}-E ; Z\right)$ with $H^{4-k}(E ; Z)$ by the Lefschetz duality. The connecting morphism $\partial: H_{1}(E ; Z) \rightarrow H_{2}\left(X_{i}-E ; Z\right)$ is then dual to the residue homomorphism; in particular, for a cycle $\gamma \in$ $H_{1}(E ; Z)$ we have 


$$
\int_{\partial r} \omega_{X_{i}}=\int_{r} \operatorname{Res}_{E} \omega_{X_{i}},
$$

where $\omega_{X_{i}} \in H^{0}\left(X_{i}, \Omega_{X_{i}}^{2}(E)\right)$ is a nowhere vanishing section induced from $\omega_{X}(i=1,2)$. Let $\{\alpha, \beta\}$ be a basis of $H_{1}(E ; Z)$. If necessary, changing $\alpha$ and $\beta$, we can normalize $\omega_{X}$ by the condition

$$
\int_{\alpha} \operatorname{Res}_{E} \omega_{X_{1}}=\tau, \operatorname{Im} \tau>0, \text { and } \int_{\beta} \operatorname{Res}_{E} \omega_{X_{1}}=1 .
$$

Now we regard ${ }^{\circ} L(X)$ as a subgroup of $\operatorname{Pic}\left(X_{1}\right) \oplus \operatorname{Pic}\left(X_{2}\right)$ under the canonical isomorphism $H^{0}\left(X_{i} ; Z\right) \cong \operatorname{Pic}\left(X_{i}\right), i=1,2$. Let c be a group homomorphism from $\operatorname{Pic}\left(X_{1}\right) \oplus \operatorname{Pic}\left(X_{2}\right)$ to Pic $(E)$ defined as follows: for $\left(\alpha_{1}, \alpha_{2}\right)$ $\in \operatorname{Pic}\left(X_{1}\right) \oplus \operatorname{Pic}\left(X_{1}\right)$,

$$
\iota\left(\left(\alpha_{1}, \alpha_{2}\right)\right)=j_{1}^{*} \alpha_{1} \otimes j_{2}^{*} \alpha_{2}^{-1},
$$

where $j_{i}$ is an inclusion $E \subset X_{i}, i=1,2$. Then, by definition, $\iota\left({ }^{\circ} L(X)\right) \subset$ $\operatorname{Pic}^{0}(E)$ (= the group of divisors of degree zero on $E$ ). So we get a group homomorphism $\iota:{ }^{0} L(X) \rightarrow \operatorname{Pic}^{0}(E)$. On the other hand, we define an AbelJacobi isomorphism

$$
\xi: \operatorname{Pic}^{0}(E) \longrightarrow J(E):=C /\{\boldsymbol{Z}+\boldsymbol{Z} \tau\}
$$

by $\xi(\gamma)=\int_{\gamma} \operatorname{Res}_{E} \omega_{X_{1}}$ for $\gamma \in \operatorname{Pic}^{0}(E)$.

We define a group homomorphism $\omega_{X}:{ }^{0} L(X) \rightarrow J(E)$ by the composite of $\iota$ and $\xi ; \omega_{X}=\xi \circ \iota$. Since $\operatorname{Res}_{E} \omega_{X_{1}}+\operatorname{Res}_{E^{\prime}} \omega_{X_{2}}=0$, the above definition is independent of selecting the component $X_{i}$ of $X$. Put $L^{*}:=\operatorname{Hom}(L, Z)$. Let $H^{+}$be the upper-half plane and $Z^{2 \times 19}$ a lattice in $L_{\vec{c}}^{*}:=L^{*} \otimes C$ which acts on $H^{+} \times L_{C}^{*}$ as follows: for $\left(\tau,\left(z_{j}\right)_{1 \leqq j \leqq 19}\right) \in H^{+} \times L_{C}^{*}$ and $\left(m_{j}^{1}, m_{j}^{2}\right)_{1 \leqq j \leqq 19} \in$ $\boldsymbol{Z}^{2 \times 19}$,

$$
\left(m_{j}^{1}, m_{j}^{2}\right):\left(\tau,\left(z_{j}\right)_{j}\right) \longrightarrow\left(\tau,\left(z_{j}+m_{j}^{1} \cdot \tau+m_{j}^{2}\right)_{j}\right) .
$$

Let $\Omega:=\left\{H^{+} \times L_{C}^{*}\right\} / Z^{2 \times 19}$ denote the quotient space. Let $\left(X, \alpha_{X}\right)$ be a marked quasi-stable $K 3$ surface with the double curve $E$. Then the period of smooth elliptic curve $E$ determines a point in $H^{+}$as usual; we denote it by $\alpha_{X}\left(\tau_{X}\right) \in H^{+}$. As mentioned above $\omega_{X}$ is now considered as a homomorphism from ${ }^{0} L(X)$ to $C$ modulo $Z+Z \alpha_{X}\left(\tau_{X}\right)$. Hence we think of $\omega_{X}$ as a homomorphism from $L$ to $C$ modulo $Z+Z \alpha_{X}\left(\tau_{X}\right)$; we denote it by $\alpha_{X}\left(\omega_{X}\right)$. In this way each marked quasi-stable $K 3$ surface $\left(X, \alpha_{X}\right)$ determines a point $\left[\left(\alpha_{X}\left(\tau_{X}\right), \alpha_{X}\left(\omega_{X}\right)\right)\right] \in \Omega$. We call $\left[\left(\alpha_{X}\left(\tau_{X}\right), \alpha_{X}\left(\omega_{X}\right)\right)\right]$ the period of $\left(X, \alpha_{X}\right)$ 
and $\Omega$ the period domain for quasi-stable $K 3$ surfaces.

(2.9) Remark. (i) The homomorphism $\omega_{X}$ coincides with the extension class of mixed Hodge structure on $X$ in the sense of Carlson's (cf. [2]).

(ii) The condition $N_{E / X_{1}} \otimes N_{E / X_{2}} \cong \mathcal{O}_{E}$ implies that $\omega_{X}\left(\left[E_{1}\right]-\left[E_{2}\right]\right) \equiv 0$ in $J(E)$. Hence if we take the quotient lattice ${ }^{0} L(X) / Z\left(\left[E_{1}\right]-\left[E_{2}\right]\right)$ for $L$, we can construct the period domain for stable $K 3$ surfaces.

(iii) We can easily check that the periods of quasi-stable $K 3$ surfaces are invariant under the modifications of type $I$ in the following sense: let $X$ be a quasi-stable $K 3$ surface with the double curve $E$ and $\phi: X \rightarrow X^{\prime}$ a modification of type I. We also think of $E$ as the double curve of $X^{\prime}$.

Then for any $\left(\left[C_{1}\right],\left[C_{2}\right]\right) \in{ }^{0} L\left(X^{\prime}\right)$,

$$
\omega_{X}\left(\phi^{*}\left(\left(\left[C_{1}\right],\left[C_{2}\right]\right)\right)\right)=\omega_{X^{\prime}}\left(\left(\left[C_{1}\right],\left[C_{2}\right]\right)\right) \text { in } J(E)
$$

(see (2.3)).

Before stating the Torelli theorem, we need some definitions. In the following, we refer to [1], [8], [9] and [14] for the reflection groups and its geometric applications.

(2.10) We keep the notation of (2.1). Let $X$ be a quasi-stable $K 3$ surface with the double curve $E$. We fix an exceptional configuration $\left\{L_{i}^{k}\right\}$ of $X$. Let $L(X)$ denote the quotient module ${ }^{0} L(X) / Z\left(\left[E_{1}\right]-\left[E_{2}\right]\right)$. Then $L(X)$ has a lattice structure induced from that of ${ }^{0} L(X)$. Moreover, by the expression of (2.6), $L(X)$ is isometric to $H \oplus\left(-E_{8}\right) \oplus\left(-E_{8}\right)$, where $H$ is the lattice of rank 2 with the corresponding matrix $\left(\begin{array}{ll}0 & 1 \\ 1 & 0\end{array}\right)$ and $E_{8}$ is the lattice of rank 8 with the Cartan matrix of the root system $E_{8}$. For brevity, we also denote the bilinear form of $L(X)$ by $\langle$,$\rangle and denote an element \left[D_{1}\right]$ $+\left[D_{2}\right] \bmod \left(\left[E_{1}\right]-\left[E_{2}\right]\right)$ of $L(X)$ by $\left[D_{1}\right]+\left[D_{2}\right]$.

We let $\Delta_{X}$ denote the set $\left\{\left[L_{1}^{9}\right]+\left[L_{2}^{9}\right],\left[L_{i}^{k}\right]-\left[L_{i}^{k+1}\right],\left[H_{i}\right]-\left[L_{i}^{1}\right]-\left[L_{i}^{2}\right]\right.$ $\left.-\left[L_{i}^{3}\right] ; \mathrm{i}=1,2, k=1,2, \cdots, 8\right\}$. As mentioned above, we regard $\Delta_{X}$ as a subset $L(X)_{R}$. Any class $\delta \in \Delta_{X}$ determines an automorphism $s_{\delta}$ of $L(X)_{R}$ defined by $s_{\delta}(x)=x+\langle x, \delta\rangle \delta$ for $x \in L(X)_{R}$. Note that $s_{\delta}$ is a reflection for the hyperplane orthogonal to $\delta$. Since the signature of $L(X)$ is $(1,17)$, the set $\left\{x \in L(X)_{R} ;\langle x, x\rangle>0\right\}$ has two connected components; write $P_{X}^{+} U$ $P_{\bar{X}}=\left\{x \in L(X)_{R} ;\langle x, x\rangle>0\right\}$. Here $P_{X}^{+}$is the component which contains an element $\left(\kappa_{1}, \kappa_{2}\right)$, where $\kappa_{i}$ is the cohomology class of the 2-form corresponding to a Kaehler metric on $X_{i}(i=1,2)$ and satisfies the condition $\left\langle\kappa_{1},\left[E_{1}\right]\right\rangle=\left\langle\kappa_{2},\left[E_{2}\right]\right\rangle$. The following result is known. 
(2.11) Proposition (cf. [14]). Let $W_{X}$ be the reflection group generated by $\Delta_{X}$ and $C_{X}$ denote the set $\left\{x \in P_{X}^{+} ;\langle x, \delta\rangle>0\right.$ for all $\left.\delta \in \Delta_{X}\right\}$. Then $W_{X}$ acts on $P_{X}^{+}$and the closure of $C_{X}$ in $P_{X}^{+}$is a fundamental domain for this action. Moreover, the Coxeter diagram of $W_{X}$ is as follows:

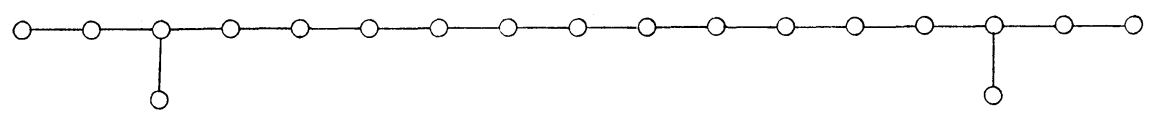

(2.12) Proposition. Let $R_{X}$ denote the set $W_{X} \cdot \Delta_{X}$. Then $R_{X}$ agrees with the set of all elements $\alpha \in L(X)$ with $\langle\alpha, \alpha\rangle=-2$.

Proof. Let $\Gamma$ be the subgroup of the group of isometries of $L(X)$ generated by the reflections $\left\{s_{\delta} ; \delta \in L(X),\langle\delta, \delta\rangle=-2\right\}$. Then $\left\{s_{\delta} ; \delta \in \Delta_{X}\right\}$ is a generator of $\Gamma$ (see [14], $\S 3$ ). Hence we have $W_{X}=\Gamma$. Let $\alpha$ be an element of $L(X)$ with $\langle\alpha, \alpha\rangle=-2$ and let denote $H_{\alpha}$ the hyperplane $\{x \in$ $\left.L(X)_{R} ;\langle x, \alpha\rangle=0\right\}$. By (2.11), we can choose $w \in W_{X}$ such that $w\left(H_{\delta}\right)=H_{\alpha}$ for some $\delta \in \Delta_{X}$. Since $H_{w(\delta)}=w\left(H_{\delta}\right)$, we have $H_{\alpha}=H_{w(\delta)}$. So $\alpha=r \cdot w(\delta)$ for some $r \in \boldsymbol{R}$. It then follows that $\alpha= \pm w(\delta)$.

(2.13) We call $C_{X}$ in (2.11) the fundamental chamber of $X$ endowed with the exceptional configuration $\left\{L_{i}^{k}\right\}$. The convex polyhedron $C_{X}$ defines the partition $R_{X}=R_{X}^{+} \Perp R_{X}^{-}$, where $R_{X}^{+}=\left\{\delta \in R_{X} ;\langle\delta, x\rangle>0\right.$ for all $\left.x \in C_{X}\right\}$. This partition has the property that

(*) If $\alpha_{1}, \cdots, \alpha_{n} \in R_{X}^{+}$, and $\alpha=\sum_{i=1}^{n} r_{i} \alpha_{i} \in R_{X}$ ( $r_{i}>0$ integers), then $\alpha \in R_{X}^{+}$(e.g. [1], p. 241).

An element $\alpha \in R_{X}$ is called nodal if either $\alpha$ is represented by a smooth rational curve with self-intersection number -2 or there is a sequence $\left\{X \underset{\phi_{1}}{\longrightarrow} X_{1} \longrightarrow \cdots \underset{\phi_{r}}{\longrightarrow} X_{r}\right\}$ of modifications of type I such that $\alpha$ is represented by $\phi_{1}^{*} \circ \ldots \circ \phi_{r}^{*}([C])$ (see (2.3)), where $C$ is a smooth rational curve on $X_{r}$ with self-intersection -2 . We denote the set of all nodal classes by $\Delta_{X}^{n}$. Let $W_{X}^{n}$ be the reflection group generated by $\Delta_{X}^{n}$ and put $R_{X}^{n}:=$ $W_{X}^{n} \cdot \Delta_{X}^{n}$. Note that if $\alpha \in R_{X}^{n} \cap \Delta_{X}$, then $\alpha$ is of one of the following types; (a) $\alpha=\left[L_{1}\right]+\left[L_{2}\right]$, where $L_{i}$ is an exceptional curve of the first kind on $X_{i}(i=1,2)$, (b) $\alpha=\left[C_{1}\right]+\cdots+\left[C_{k}\right](k \geqq 1)$, where $C_{i}$ is a smooth rational curve with self-intersection -2 and $\left(C_{i}, C_{i+1}\right)=1,\left(C_{i}, C_{j}\right)=0$ for $i \neq j \pm 1$. Moreover, in Section 3, we shall characterize $R_{X}^{n}$ as follows (see 3.4)): $R_{X}^{n}=\left\{\alpha \in R_{X} ; \omega_{X}(\alpha)=0\right.$ in $\left.J(E)\right\}$. Let $C_{X}^{n}$ be the set $\left\{x \in P_{X}^{+} ;\langle x, \delta\rangle>0\right.$ for all $\left.\delta \in \Delta_{X}^{n}\right\}$. Then Proposition (2.11) holds for the action of $W_{X}^{n}$ on $P_{X}^{+}$ 
and a fundamental domain $C_{X}^{n}$. We remark that $C_{X}^{n}$ is independent of the choice of an exceptional configuration of $X$. We call $C_{X}^{n}$ the nodal chamber of $X$. Now we formulate our main results.

(2.14) Theorem. Let $X=X_{1} \cup X_{2}$ and $X^{\prime}=X_{1}^{\prime} \cup X_{2}^{\prime}$ be two quasi-stable $K 3$ surfaces of type II with the double curves $E, E^{\prime}$, respectively. Let $\phi^{*}$ : ${ }^{0} L\left(X^{\prime}\right) \oplus{ }^{0} W_{1}\left(X^{\prime}\right) \rightarrow{ }^{0} L(X) \oplus{ }^{0} W_{1}(X)$ be an isometry such that (i) $\phi^{*}\left({ }^{0} W_{1}\left(X^{\prime}\right)\right)$ $={ }^{0} W_{1}(X)$, (ii) $\phi^{*}\left(\left[E_{1}^{\prime}\right]-\left[E_{2}^{\prime}\right]\right)= \pm\left(\left[E_{1}\right]-\left[E_{2}\right]\right)\left(B y\right.$ (ii), $\phi^{*}$ induces an isometry from $L\left(X^{\prime}\right)$ to $L(X)$. For simplicity, we also denote it by $\phi^{*}$ ), (iii) $\phi^{*}\left(P_{X^{\prime}}^{+}\right)=P_{X}^{+}$and $\phi^{*}\left(C_{X^{\prime}}^{n}\right)=C_{X}^{n}$, (iv) $\phi^{*}$ sends $H^{1,0}\left(E^{\prime}, C\right)$ to $H^{1,0}(E, C)$ and $\omega_{X}\left(\phi^{*}\left(\left(\alpha_{1}, \alpha_{2}\right)\right)\right)=J\left(\phi^{*}\right)\left(\omega_{X^{\prime}}\left(\left(\alpha_{1}, \alpha_{2}\right)\right)\right)($ in $J(E))$ for $\left(\alpha_{1}, \alpha_{2}\right) \in{ }^{0} L\left(X^{\prime}\right)$, where $J\left(\phi^{*}\right)$ is the isomorphism of Jacobian varieties induced from $\phi^{*}: H^{1,0}\left(E^{\prime}\right) \rightarrow H^{1,0}(E)$. Then there is a sequence

$$
\left\{X \underset{\phi_{1}}{\longrightarrow} X_{1} \underset{\phi_{2}}{\rightarrow} \cdots \longrightarrow X_{r-1} \underset{\phi_{r}}{\longrightarrow} X_{r}\right\}
$$

of modifications of type I and an isomorphism $\psi: X_{r} \rightarrow X^{\prime}$ such that the associated isometry

$$
\phi_{1}^{*} \circ \cdots \circ \phi_{r}^{*} \circ \psi^{*}:{ }^{0} L\left(X^{\prime}\right) \oplus{ }^{0} W_{1}\left(X^{\prime}\right) \longrightarrow{ }^{0} L(X) \oplus{ }^{0} W_{1}(X)
$$

agrees with $\phi^{*}$.

(2.15) Theorem. For every point $[(\tau, \omega)] \in \Omega$, there is a marked quasistable K3 surface of type II with the period $[(\tau, \omega)]$.

Proofs of (2.14), (2.15) will be given in Section 3.

(2.16) Remark. Let $D$ be the period domain for smooth $K 3$ surfaces. Let us recall that there is an étale covering $\tilde{D} \rightarrow D$ such that $\tilde{D}$ is a relevant moduli space for marked Kaehler $K 3$ surfaces ([1], p. 239, or [10], Theorem (10.5)).

In our case, the corresponding situation is as follows: We let $N$ denote the lattice $H \oplus\left(-E_{8}\right) \oplus\left(-E_{8}\right)$. As remarked in (2.9), (ii), we can construct the period domain $\Omega_{0}:=\left\{H^{+} \times N_{C}\right\} / Z^{2 \times 18}$ for stable $K 3$ surfaces by the same way for $\Omega$. Here we select the lattice $Z^{2 \times 18} \subset N_{C}$ which contains $N$. Let $W$ be the reflection group generated by $\Delta:=\{\delta \in N ;\langle\delta, \delta\rangle=-2\}$ and consider the space $\Omega_{0}^{\prime}$ consisting of pairs $([(\tau, \omega)], \kappa) \in \Omega_{0} \times N_{R}$ satisfying $\langle\kappa, \kappa\rangle>0$. Naturally $W$ acts on $\Omega_{0}^{\prime}$ : for $\delta \in \Delta$,

$$
s_{\hat{\delta}}:([(\tau, \omega)], \kappa) \longrightarrow\left(\left[\left(\tau, s_{\delta}(\omega)\right)\right], s_{\delta}(\kappa)\right) .
$$

Let $\Omega_{0}^{\prime \prime} \subset \Omega_{0}^{\prime}$ denote the complement of the set of fixed points of reflections. 
We define an equivalence relation $\sim$ on $\Omega_{0}^{\prime \prime}$ by letting $([(\tau, \omega)], \kappa) \sim\left(\left[\left(\tau^{\prime}\right.\right.\right.$, $\left.\left.\left.\omega^{\prime}\right)\right], \kappa^{\prime}\right)$ if and only if $[(\tau, \omega)]=\left[\left(\tau^{\prime}, \omega^{\prime}\right)\right]$ and $\kappa$ and $\kappa^{\prime}$ belong to the same connected component of $\Omega_{0}^{\prime \prime} \cap\left([(\tau, \omega)] \times N_{R}\right)$. Let $\tilde{\Omega}_{0}:=\tilde{\Omega}_{0}^{\prime \prime} / \sim$ denote the quotient space. It is provided with a canonical projection

$$
\pi: \tilde{\Omega}_{0} \longrightarrow \Omega_{0} \text {. }
$$

Then $\widetilde{\Omega}_{0}$ receives the structure of analytic space, étale over $\Omega_{0}$ ([10], Lemma (10.4)).

Let $M$ be the set of isomorphism classes of marked stable $K 3$ surfaces (with isomorphisms defined in the obvious manner). Then we associate a map $p: M \rightarrow \tilde{\Omega}_{0}$ which assigns to the isomorphism class of marked stable $K 3$ surface $\left(X, \alpha_{X}\right)$ the equivalence class of $\left(\left(\alpha_{X}\left(\tau_{X}\right), \alpha_{X}\left(\omega_{X}\right)\right)\right.$, the nodal chamber of $X)$. Here we use the following fact which will be proved in Section 3, Proposition (3.4); If $\delta \in R_{X}$, then $\delta \in R_{X}^{n}$ if and only if $\omega_{X}(\delta) \equiv 0$ in $J(E)$.

In this situation we reformulate theorems (2.14), (2.15) as follows:

(i) The map $p: M \rightarrow \tilde{\Omega}_{0}$ is surjective.

(ii) Let $\left(X, \alpha_{X}\right)$ and $\left(X^{\prime}, \alpha_{X^{\prime}}\right)$ be two marked stable $K 3$ surfaces whose images by the map $p$ are contained in the same fibre of $\pi$, then there is a bimeromorphic map $X \longrightarrow X^{\prime}$ which is a composite of modifications of type I.

\section{$\S 3 . \quad$ Proofs of (2.14), (2.15)}

Let $X=X_{1} \cup X_{2}$ be a quasi-stable $K 3$ surface and $E$ the double curve. We fix an exceptional configuration $\left\{L_{i}^{k}\right\}$ of $X$. First we shall prove the following two lemmas. We keep the notation in Section 2.

(3.1) Lemma. If $\alpha \in \Delta_{X}-R_{X}^{n}$, then either $\left\{s_{\alpha}\left(\left[L_{i}^{k}\right]\right)\right\}$ is an exceptional configuration of $X$ or there is a composition $\left\{\phi:=\phi_{1} \circ \phi_{2}: X^{\prime} \underset{\phi_{2}}{\rightarrow} X^{\prime \prime} \underset{\phi_{1}}{\longrightarrow} X\right\}$ of modifications of type I such that $\left\{\phi^{*} \circ s_{\alpha}\left(\left[L_{i}^{k}\right]\right)\right\}$ is an exceptional configuration of $X^{\prime}$. (We remark here that every reflection $s_{\alpha}, \alpha \in \Delta_{X}$, is defined on $H^{2}\left(X_{1} ; R\right) \oplus H^{2}\left(X_{2} ; R\right)$, and the expression $s_{\alpha}\left(\left[L_{\imath}^{k}\right]\right)$ is in this meaning. $)$

(3.2) Lemma. If $w \in W_{X}$ is such that $w\left(C_{X}\right) \subset C_{X}^{n}$, then either $\left\{w\left(\left[L_{i}^{k}\right]\right)\right\}$ is also an exceptional configuration of $X$ or there is a composition $\left\{X_{r} \overrightarrow{\phi_{r}}\right.$ $\left.X_{r-1} \underset{\phi_{r-1}}{\rightarrow} \cdots \longrightarrow X_{1} \underset{\phi_{1}}{\rightarrow} X\right\}$ of modifications of type I such that $\left\{\phi_{r}^{*} \circ \cdots \circ\right.$ $\left.\phi_{1}^{*} \circ w\left(\left[L_{i}^{k}\right]\right)\right\}$ is an exceptional configuration of $X_{1}$.

(3.3) Remark. Looijenga [9] has deeply studied rational surfaces with 
anti-canonical cycle. Our process in the above lemmas is similar to his method, but in our case modifications of type I occur, which make the argument more complicated (see [9], §4).

Proof of (3.1) (see [9], §3). Let $\alpha$ be an element of $\Delta_{X}$. If $\alpha=\left[L_{i}^{k-1}\right]$ - $\left[L_{i}^{k}\right]$, then $\alpha \notin R_{X}^{n}$ if and only if $L_{i}^{k}$ is not contained in $L_{i}^{k-1}$, hence $L_{i}^{k}$ and $L_{i}^{k-1}$ are disjoint (see (2.13)). Since $s_{\alpha}$ interchanges $L_{i}^{k-1}$ and $L_{i}^{k}$ and leaves all other $L_{i}^{k^{\prime}}$ fixed, everything is obvious in this case. Next if $\alpha=\left[H_{i}\right]$ $-\left[L_{i}^{1}\right]-\left[L_{i}^{2}\right]-\left[L_{i}^{3}\right]$, then the condition that $\alpha \notin R_{X}^{n}$ implies that $\pi_{i}\left(L_{i}^{1}\right), \pi_{i}\left(L_{i}^{2}\right)$, $\pi_{i}\left(L_{i}^{3}\right)$ are not collinear. (Here $\pi_{i}: X_{i} \rightarrow \bar{X}_{i}$ is a relatively minimal model of $X_{i}$ (see (2.5)).) Suppose that $\pi_{i}\left(H_{i}\right)$ is not a tangent line of $E$. Then $\pi_{i}\left(L_{i}^{1}\right), \pi_{i}\left(L_{i}^{2}\right)$ and $\pi_{i}\left(L_{i}^{3}\right)$ are distinct. Moreover, by the assumption of the indices of the exceptional configuration, each $L_{i}^{k}$ is a maximal exceptional curve in the sense that $L_{i}^{k}=\pi_{i}^{-1} \circ \pi_{i}\left(L_{i}^{k}\right)$. Now $s_{\alpha}\left(\left[L_{i}^{1}\right]\right)=\left[H_{i}\right]-\left[L_{i}^{2}\right]-\left[L_{i}^{3}\right]$ is represented by the total transform of the line $\bar{X}_{i}=\boldsymbol{P}^{2}$ which passes through $\pi_{i}\left(L_{i}^{2}\right)$ and $\pi_{i}\left(L_{i}^{3}\right)$ minus $L_{i}^{2}+L_{i}^{3}$. If we denote this representative ' $L_{i}^{1}$ (and ' $L_{i}^{2}$, resp. ' $L_{i}^{3}$, the corresponding representatives of $s_{\alpha}\left(\left[L_{i}^{2}\right]\right)$, resp. $\left.s_{\alpha}\left(\left[L_{i}^{3}\right]\right)\right)$, then it is clear that ${ }^{\prime} L_{i}^{1},{ }^{\prime} L_{i}^{2},{ }^{\prime} L_{i}^{3}$ are disjoint and that any $L_{i}^{\mu}(\mu>3)$ which meets ' $L_{i}^{k}(1 \leqq k \leqq 3)$ is actually contained in ' $L_{i}^{k}$. So $\left\{s_{\alpha}\left(\left[L_{i}^{k}\right]\right)\right\}$ is an exceptional configuration of $X$. The proof for the case that $\pi_{i}\left(H_{i}\right)$ is a tangent line is similar.

Last of all, if $\alpha=\left[L_{1}^{9}\right]+\left[L_{2}^{9}\right]$, the condition that $\alpha \notin R_{X}^{n}$ just means that the points $L_{1}^{9} \cap E$ and $L_{2}^{9} \cap E$ are distinct. Note that $s_{\alpha}\left(\left[L_{1}^{9}\right]+\left[L_{2}^{9}\right]\right)=$ $-\left[L_{1}^{9}\right]-\left[L_{2}^{9}\right], \quad s_{\alpha}\left(\left[L_{1}^{8}\right]-\left[L_{1}^{9}\right]\right)=\left[L_{1}^{8}\right]+\left[L_{2}^{9}\right], \quad s_{\alpha}\left(\left[L_{2}^{8}\right]-\left[L_{2}^{9}\right]\right)=\left[L_{2}^{8}\right]+\left[L_{1}^{9}\right]$ and $s_{\alpha}\left(\left[L_{i}^{k}\right]\right)=\left[L_{i}^{k}\right]$ for $i=1,2,1 \leqq k \leqq 8$. Let $\phi: X^{\prime} \longrightarrow X$ be the birational map obtained by the modifications of type I along the exceptional curves of the first kind $L_{1}^{9}, L_{2}^{9}$ (by the assumption of indices of $\left\{L_{i}^{k}\right\}, L_{1}^{9}$ and $L_{2}^{9}$ are first kind). Let ' $L_{1}^{9}$ (resp. ' $L_{2}^{9}$ ) be the exceptional curve on $X^{\prime}$ obtained by blowing up the point $L_{1}^{9} \cap E$ (resp. $L_{2}^{9} \cap E$ ).
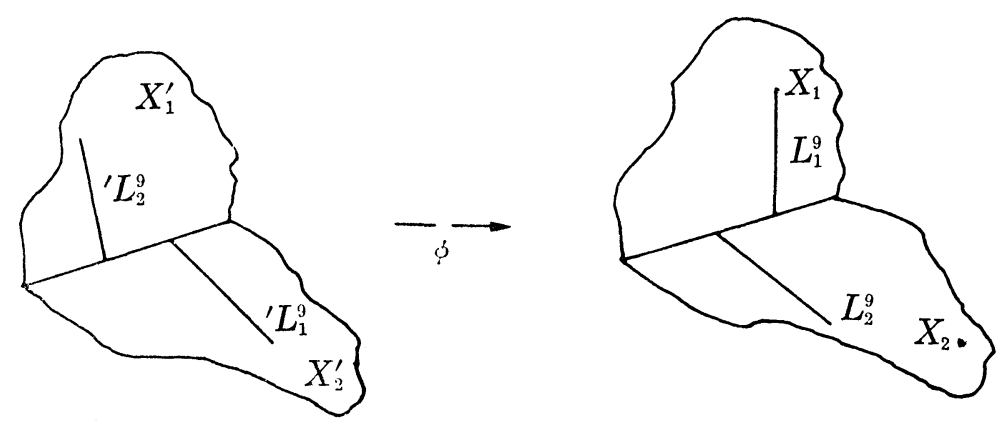
Put $\left[{ }^{\prime} L_{i}^{k}\right]=\phi^{*}\left(\left[L_{i}^{k}\right]\right), i=1,2,1 \leqq k \leqq 8$, Then we have that $\phi^{*}\left(-\left[L_{1}^{9}\right]\right.$ $\left.\left.\left.-\left[L_{2}^{9}\right]\right)={ }^{\prime} L_{1}^{9}\right]+{ }^{\prime} L_{2}^{9}\right], \quad \phi^{*}\left(\left[L_{1}^{8}\right]+\left[L_{2}^{9}\right]\right)=\left[{ }^{\prime} L_{1}^{8}\right]-\left[{ }^{\prime} L_{2}^{9}\right]$ and $\phi^{*}\left(\left[L_{2}^{8}\right]+\left[L_{1}^{9}\right]\right)=$ $\left[{ }^{\prime} L_{2}^{8}\right]-\left[{ }^{\prime} L_{1}^{9}\right]$. Now it is easily check that $\left.\left\{{ }^{\prime} L_{i}^{k}\right]\right\}$ is an exceptional configuration. We leave the proof to the reader.

Proof of Lemma (3.2) (see [9], (3.5), (4.2)). First we claim that $C_{X}^{n}$ contains $C_{X}$. For this purpose, it is sufficient to prove that $\Delta_{X}^{n}$ is contained in $R_{X}^{+}$(see (2.13)). Let $\delta=[D]\left(\in \Delta_{X}^{n}\right)$ be a nodal class. If $D$ has a component which is not contained in $L_{i}^{k}$, then obviously $\left.\left\langle\delta,\left[H_{1}\right]+\left[H_{2}\right]\right\rangle\right\rangle 0$. Since $\left[H_{1}\right]+\left[H_{2}\right] \in \bar{C}_{X}, \delta$ is contained in $R_{X}^{+}$. Now we assume that $\delta$ is represented by a divisor $D$ whose each irreducible component is contained in some $L_{i}^{k}$. Since $D$ is connected (by definition), $D$ is one of the following two types: (i) $D$ is a smooth rational curve with self-intersection -2, (ii) $D=C_{1}+C_{2}$, where $C_{i}$ is an exceptional curve of the first kind on $X_{i}(i=1,2)$ with $C_{1} \cap E=C_{2} \cap E$. If $D$ is a smooth rational curve with self-intersection -2 , then $D$ is represented by $L_{i}^{k}-L_{i}^{k^{\prime}}\left(1 \leqq k<k^{\prime} \leqq 9\right)$. Hence $[D]$ is a positive linear combination of elements of $\Delta_{X}$ :

$$
[D]=\left(\left[L_{i}^{k}\right]-\left[L_{i}^{k+1}\right]\right)+\cdots+\left(\left[L_{i}^{k^{\prime}-1}\right]-\left[L_{i}^{k^{\prime}}\right]\right) .
$$

By (2.13), (*), $[D]$ is contained in $R_{X}^{+}$.

Next, if $D=C_{1}+C_{2}$, where $C_{i}$ is an exceptional curve of the first kind on $X_{i}(i=1,2)$ with $C_{1} \cap E=C_{2} \cap E$, then $\left[C_{1}\right]+\left[C_{2}\right]$ is a positive linear combination of elements of $\Delta_{X}$ :

$$
\begin{aligned}
{\left[C_{1}\right]+\left[C_{2}\right]=} & \left(\left[L_{1}^{j}\right]-\left[L_{1}^{j+1}\right]\right)+\cdots+\left(\left[L_{1}^{8}\right]-\left[L_{1}^{9}\right]\right)+\left(\left[L_{1}^{9}\right]+\left[L_{2}^{9}\right]\right) \\
& +\left(\left[L_{2}^{8}\right]-\left[L_{2}^{9}\right]\right)+\cdots+\left(\left[L_{2}^{k}\right]-\left[L_{2}^{k+1}\right]\right) .
\end{aligned}
$$

So in this case $\left[C_{1}\right]+\left[C_{2}\right] \in R_{X}^{+}$, too. Hence $C_{X}$ is contained in $C_{X}^{n}$. Note that there are no hyperplane separating $C_{X}$ from $w\left(C_{X}\right)$ which is orthogonal to some $\alpha \in \Delta_{X}^{n}$.

Now we prove (3.2). We pick a point $x_{0} \in w\left(C_{X}\right)$ and denote the set $\left\{\alpha \in R_{X}^{+} ;\left\langle\alpha, x_{0}\right\rangle<0\right\}$ by $\Phi_{x_{0}}$. Then $\Phi_{x_{0}}$ corresponds to the set of hyperplanes orthogonal to $\alpha \in R_{X}$ which separate $C_{X}$ and $w\left(C_{X}\right)$. By [15] Lemma 9 (in $\S 3$ ), $\Phi_{x_{0}}$ is a finite set. Hence we can index the elements of $\Phi_{x_{0}}$ as follows: $\Phi_{x_{0}}=\left\{\alpha_{1}, \cdots, \alpha_{k}\right\}$ (with $k=\operatorname{card} \Phi_{x_{0}}$ ) such that the set

$$
\left\{C_{i}=s_{\alpha_{i}} \circ \cdots \circ s_{\alpha_{1}}\left(C_{X}\right) ; i=1, \cdots, k\right\}
$$

is a chain of the fundamental chambers from $C_{X}$ to $w\left(C_{X}\right)$; more precisely the intersection of $C_{i-1}$ and $C_{i}=s_{\alpha_{i}}\left(C_{i-1}\right)$ is a non-empty open set in the hyperplane $H_{\alpha_{i}}=\left\{x \in L(X)_{R} ;\left\langle x, \alpha_{i}\right\rangle=0\right\}$. Note that $\alpha_{1}$ is contained in 
$\Delta_{X}$. Since $C_{X}$ is contained in $C_{X}^{n}$, the condition that $\alpha_{i} \in R_{X}^{+}$implies that $\alpha_{i} \notin R_{X}^{n}(i=1, \cdots, k)$. With induction on $i$, Lemma (3.2) now follows easily from (3.1).

Proof of Theorem (2.14). Let $\left\{L_{i}^{k}\right\}$ (resp. $\left\{{ }^{\prime} L_{i}^{k}\right\}$ ) be an exceptional configuration of $X$ (resp. $X^{\prime}$ ). Let $C_{X}$ (resp. $C_{X^{\prime}}$ ) be the fundamental chamber of $X$ (resp. $X^{\prime}$ ) endowed with the exceptional configuration $\left\{L_{i}^{k}\right\}$ (resp. $\left.\left\{{ }^{\prime} L_{i}^{k}\right\}\right)$. By the assumptions in (2.14), we have that $\phi^{*}\left(P_{X^{\prime}}^{+}\right)=P_{X}^{+}, \phi^{*}\left(C_{X^{\prime}}^{n}\right)$ $=C_{X}^{n}$. On the other hand, by (2.12), we have $\phi^{*}\left(R_{X^{\prime}}\right)=R_{X}$. Hence both $C_{X}$ and $\phi^{*}\left(C_{X^{\prime}}\right)$ are fundamental domains for the action of $W_{X}$ on $P_{X}^{+}$(see (2.11)). In particular, $w\left(C_{X}\right)=\phi^{*}\left(C_{X^{\prime}}\right)$ for some $w \in W_{X}$. It then follows that $w\left(\Delta_{X}\right)=\phi^{*}\left(\Delta_{X^{\prime}}\right)$. Hence, if necessary, changing the indices of $X_{1}$ and $X_{2}$ (or equivalently, replacing $\phi^{*}$ by $\iota \circ \phi^{*}$, where $\iota$ is the symmetry of the Coxeter diagram of $W_{X}$ (see (2.11)), we can assume that

$$
\begin{aligned}
& w\left(\left[L_{i}^{k}\right]-\left[L_{i}^{k+1}\right]\right)=\phi^{*}\left(\left[{ }^{\prime} L_{i}^{k}\right]-\left[{ }^{\prime} L_{i}^{k+1}\right]\right), \\
& w\left(\left[L_{1}^{9}\right]+\left[L_{2}^{9}\right]\right)=\phi^{*}\left(\left[{ }^{\prime} L_{1}^{9}\right]+\left[{ }^{\prime} L_{2}^{9}\right]\right) \quad \text { and } \\
& w\left(\left[H_{i}\right]-\left[L_{i}^{1}\right]-\left[L_{i}^{2}\right]-\left[L_{i}^{3}\right]\right)=\phi^{*}\left(\left[{ }^{\prime} H_{i}\right]-\left[{ }^{\prime} L_{i}^{1}\right]-\left[{ }^{\prime} L_{i}^{2}\right]-\left[{ }^{\prime} L_{i}^{3}\right]\right), \\
& \quad i=1,2, \quad k=1,2, \cdots, 8 .
\end{aligned}
$$

Since $w\left(C_{X}\right)$ is contained in $\phi^{*}\left(C_{X}^{n}\right)=C_{X}^{n}$, by applying Lemma (3.2), we get a sequence $\left\{X_{r} \underset{\phi_{r}}{\rightarrow} X_{r-1} \longrightarrow \cdots \rightarrow X_{1} \underset{\phi_{1}}{\rightarrow} X_{0}=X\right\}$ of modifications of type I such that $\left\{\phi_{r}^{*} \circ \cdots \circ \phi_{1}^{*} \circ w\left(\left[L_{i}^{k}\right]\right)\right\}$ is an exceptional configuration of $X_{r}=X_{1, r} \cup X_{2, r}$. We denote $\phi_{r}^{*} \circ \cdots \circ \phi_{1}^{*} \circ \phi^{*}$ by $\psi^{*}$ and $\left\{\phi_{r}^{*} \circ \cdots \circ \phi_{1}^{*}\right.$ 。 $\left.w\left(\left[L_{i}^{k}\right]\right)\right\}$ by $\left\{\left[L_{i, r}^{k}\right]\right\}$. Let $\pi_{i, r}: X_{i, r} \rightarrow \bar{X}_{i, r}$ be a relatively minimal model and let $E_{r}$ be the double curve of $X_{r}$. Then we have that

$$
\begin{aligned}
& \psi^{*}\left(\left[{ }^{\prime} L_{i}^{k}\right]-\left[{ }^{\prime} L_{i}^{k+1}\right]\right)=\left[L_{i, r}^{k}\right]-\left[L_{i, r}^{k+1}\right], \\
& \psi^{*}\left(\left[{ }^{\prime} L_{1}^{9}\right]+\left[{ }^{\prime} L_{2}^{9}\right]\right)=\left[L_{1, r}^{9}\right]+\left[L_{2, r}^{9}\right] \text { and } \\
& \psi^{*}\left(\left[{ }^{\prime} H_{i}\right]-\left[{ }^{\prime} L_{i}^{1}\right]-\left[{ }^{\prime} L_{i}^{2}\right]-\left[{ }^{\prime} L_{i}^{3}\right]\right)=\left[H_{i, r}\right]-\left[L_{i, r}^{1}\right]-\left[L_{i, r}^{2}\right]-\left[L_{i, r}^{3}\right] \\
& \quad(i=1,2, k=1,2, \cdots, 8),
\end{aligned}
$$

where $H_{i, r}$ is the total transform of the line in $\bar{X}_{i, r}=\boldsymbol{P}^{2}$ which passes through $\pi_{i, r}\left(L_{i, r}^{1}\right)$ and $\pi_{i, r}\left(L_{i, r}^{2}\right)$ (at least when $\pi_{i, r}\left(L_{i, r}^{1}\right) \neq \pi_{i, r}\left(L_{i, r}^{2}\right)$; otherwise take the tangent line of $\pi_{i, r}\left(E_{r}\right)$ at $\pi_{i, r}\left(L_{i, r}^{1}\right)=\pi_{i, r}\left(L_{i, r}^{2}\right)$ ). Let $u_{i}^{k}$ (resp. ' $u_{i}^{k}$ ) be the point $E_{r} \cap L_{i, r}^{k}$ (resp. $\left.E^{\prime} \cap{ }^{\prime} L_{i}^{k}\right)$. Then by the equations $\psi^{*}\left(\left[L_{1}^{k}\right]-\right.$ $\left.\left[{ }^{\prime} L_{1}^{k+1}\right]\right)=\left[L_{1, r}^{k}\right]-\left[L_{1, r}^{k+1}\right]$ and the assumption (iv) in (2.14), there is an isomorphism

$$
\psi_{0}: E_{r} \longrightarrow E^{\prime}
$$


such that $\psi_{0}\left(u_{1}^{k}\right)={ }^{\prime} u_{1}^{k}(k=1, \cdots, 9)$. Moreover, fron $\psi^{*}\left(\left[L_{1}^{9}\right]+\left[{ }^{\prime} L_{2}^{9}\right]\right)=$ $\left[L_{1, r}^{9}\right]+\left[L_{2, r}^{9}\right]$, we obtained

$$
\psi_{0}\left(u_{1}^{9}\right)-\psi_{0}\left(u_{2}^{9}\right)={ }^{\prime} u_{1}^{9}-{ }^{\prime} u_{2}^{9}
$$

(Here we consider $E_{r}$ (resp. $E^{\prime}$ ) a group with the identity element $u_{1}^{1}$ (resp. $\left.\left.' u_{1}^{1}\right)\right)$. So we get $\psi_{0}\left(u_{2}^{9}\right)={ }^{\prime} u_{2}^{9}$. By the equations $\psi^{*}\left(\left[{ }^{\prime} L_{2}^{k}\right]-\left[{ }^{\prime} L_{2}^{k+1}\right]\right)=\left[L_{2, r}^{k}\right]$ - $\left[L_{2, r}^{k+1}\right]$, we conclude that $\psi_{0}\left(u_{i}^{k}\right)={ }^{\prime} u_{i}^{k}(i=1,2, k=1,2, \cdots, 9)$.

Let $u_{i}$ (resp. ' $u_{i}$ ) denote the third point at which $H_{i, r}$ intersects $E_{r}$ (resp. ' $H_{i}$ intersects $E^{\prime}$ ). Then the linear system $\left|u_{i}+u_{i}^{1}+u_{i}^{2}\right|$ (resp. $\mid u_{i}+$ ' $\left.u_{i}^{1}+{ }^{\prime} u_{i}^{2}\right)$ gives an embedding $E_{r} \rightarrow \boldsymbol{P}^{2}=\bar{X}_{i, r}$ (resp. $\left.E^{\prime} \rightarrow \boldsymbol{P}^{2}=\bar{X}_{i}^{\prime}\right), i=1,2$. By the above equation w.r.t. $\psi^{*}$, it follows that

$$
\psi_{0}^{*}\left|{ }^{\prime} u_{i}+{ }^{\prime} u_{i}^{1}+{ }^{\prime} u_{i}^{2}\right|=\left|u_{i}+u_{i}^{1}+u_{i}^{2}\right| \quad(i=1,2) .
$$

Hence $\psi_{0}$ can extend to an isomorphism $\bar{\psi}_{i}: \bar{X}_{i, r} \rightarrow \bar{X}_{i}^{\prime}(i=1,2)$. Obviously $\bar{\psi}_{i}$ induces an isomorphism $\psi_{i}: X_{i, r} \rightarrow X_{i}^{\prime}(i=1,2)$. Moreover $\psi:=\psi_{1} \cup \psi_{2}$ : $X_{1, r} \cup X_{2, r} \rightarrow X_{1}^{\prime} \cup X_{2}^{\prime}$ is an isomorphism and by construction, $\psi^{*}=\phi_{r}^{*} \circ \cdots 。$ $\phi_{1}^{*} \circ \phi^{*}$ agrees with an isomorphism induced from $\psi$.

Proof of Theorem (2.15). Let $[(\tau, \omega)] \in \Omega$ be given. Let $E$ be a smooth elliptic curve with the period $\{1, \tau\}$ and $\omega_{E}$ a holomorphic 1-form on $E$ such that

$$
\int_{\alpha} \omega_{E}=\tau, \quad \int_{\beta} \omega_{E}=1
$$

for a suitable basis $\{\alpha, \beta\}$ of $H_{1}(E ; Z)$. We regard a basis of $L$ as a coordinate system of $L_{c}$ and write

$$
\omega=\left[\left(t_{1}, t_{2}, \cdots, t_{19}\right)\right] \in L_{C}^{*} / Z^{2 \times 19},
$$

where $\left(t_{1}, t_{2}, \cdots, t_{19}\right) \in L_{c}^{*}$. Now we consider the following equations in the points $z_{\mu, j}, \mu=1,2, j=0,1, \cdots, 9$, on $E$ : modulo $Z+Z z$,

$$
\begin{aligned}
& \text { (i) } \sum_{j=1}^{9} \int_{z_{1, j}}^{z_{1,0}} \omega_{E} \equiv t_{1}, \quad \sum_{j=1}^{9} \int_{z_{2,0}}^{z_{2, j}} \omega_{E} \equiv t_{2} \\
& \text { (ii) } \int_{z_{2,9}}^{z_{1,9}} \omega_{E} \equiv t_{3} \\
& \text { (iii) } \quad \sum_{j=1}^{3} \int_{z_{1, i}}^{z_{1,0}} \omega_{E} \equiv t_{4}, \quad \int_{z_{1, i+1}}^{z_{1, i}} \omega_{E} \equiv t_{i+4}(i=1, \cdots, 7) \\
& \text { (iv) } \quad \sum_{j=1}^{3} \int_{z_{2,0}}^{z_{2, j}} \omega_{E} \equiv t_{12}, \quad \int_{z_{2, i}}^{z_{2, i+1}} \omega_{E} \equiv t_{i+12}(i=1, \cdots, 7) .
\end{aligned}
$$

These equations are correspond to the expression (2.6) of the basis of ${ }^{\circ} \mathrm{L}$. By Jacobi's inversion theorem, we can solve these equations as follows: 
First we take a point $z_{1,0}=p_{0} \in E$, arbitrarily. Applying Jacobi theorem on the equation (iii), we can find $p_{1}, \cdots, p_{8} \in E$ such that $\left\{z_{1, i}=p_{i} ; i=\right.$ $1, \cdots, 8\}$ satisfies the equation (iii). Next from the equation (i), we can find a point $p_{9} \in E$ such that $\left\{z_{1, i}=p_{i} ; i=1, \ldots, 8,9\right\}$ satisfies the first of the equation (i). Similarly from the equation (ii), there is a point $q_{9} \in E$ such that

$$
\int_{q_{9}}^{p_{9}} \omega_{E} \equiv t_{3} \bmod Z+Z \tau
$$

Moreover, by the relations

$$
\begin{aligned}
& \left(z_{1,1}+\cdots+z_{1,9}\right)-\left(z_{2,1}+\cdots+z_{2,9}\right) \\
& \quad=9\left(z_{1,9}-z_{2,9}\right)+\sum_{i=1}^{8} i\left(z_{1, i}-z_{1, i+1}\right)+\sum_{i=1}^{8} i\left(z_{2, i+:}-z_{2, i}\right)
\end{aligned}
$$

and

$$
\begin{aligned}
9 z_{i, 0}- & z_{i, 1}-\cdots-z_{i, 9} \\
= & 3\left(3 z_{i, 0}-z_{i, 1}-z_{i, 2}-z_{i, 3}\right)+2\left(z_{i, 1}-z_{\imath, 2}\right)+4\left(z_{i, 2}-z_{i, 3}\right) \\
& +\sum_{k=1}^{6}(7-k)\left(z_{i, k+2}-z_{i, k+3}\right),
\end{aligned}
$$

we can write that

$$
\int_{z_{2,0}}^{z_{1,0}} \omega_{E} \equiv \text { a linear combination of }\left\{t_{1}, t_{2}, \cdots, t_{19}\right\} .
$$

Again, applying Jacobi theorem on this equation and the equation (iv), we can find $q_{0}, q_{1}, \cdots, q_{8} \in E$ such that $\left\{z_{2, j}=q_{j} ; 0 \leqq j \leqq 9\right\}$ is a solution of the equation (iv) and the second of the equation (i). Consequently we obtain the solution $\left\{z_{1, i}=p_{i}, z_{2, j}=q_{j} ; 0 \leqq i, j \leqq 9\right\}$ of the equations (i)(iv).

Let $\bar{X}_{i}$ be a copy of $\boldsymbol{P}^{2}(i=1,2)$. Now we consider the embeddings $\left|3 p_{0}\right|: E \rightarrow \bar{X}_{1}=\boldsymbol{P}^{2},\left|3 q_{0}\right|: E \rightarrow \bar{X}_{2}=\boldsymbol{P}^{2}$. Let $X_{1}$ (resp. $X_{2}$ ) be the surface obtained from $\bar{X}_{1}$ (resp. $\bar{X}_{2}$ ) by taking successive blowing ups at $p_{1}, \cdots, p_{9}$ (resp. $q_{1}, \cdots, q_{9}$ ). Let $E_{1}$ (resp. $E_{2}$ ) be the proper transform of $E$ by the above blowing ups. We denote the induced isomorphism from $E_{1}$ to $E_{2}$ by $\phi$. Then the surface $X$ obtained from $X_{1}$ and $X_{2}$ by patching through $E_{1}$ and $E_{2}$ under the isomorphism $\phi$ is the required one.

Lastly we prove the following proposition which has been used in the reformulation of theorems (2.14), (2.15) (see (2.16)).

For $\alpha \in \Delta_{X}$, we can define $\omega_{X}(\alpha)$ by regarding $\alpha$ as an element in ${ }^{\circ} L(X)$. We can also define $\omega_{X}(\alpha)$ for $\alpha \in R_{X}$, since $\alpha$ is represented by an element of $Z \cdot \Delta_{X}$. 
(3.4) Proposition. If $\alpha \in R_{X}$, then $\alpha \in R_{X}^{n}$ if and only if $\omega_{X}(\alpha)=0$ in $J(E)$.

Proof. Let $\delta\left(\in \Delta_{X}^{n}\right)$ be a nodal class. Then, by definition and (2.9), (iii), $\omega_{X}(\delta)=0$ in $J(E)$. If $\alpha \in R_{X}^{n}$, then $\alpha \equiv 0 \bmod Z \cdot \Delta_{X}^{n}$ and so $\omega_{X}(\alpha)=0$ in $J(E)$. Conversely, if $\alpha \in R_{X}$ such that $\omega_{X}(\alpha)=0$ in $J(E)$, then $\alpha=w(\beta)$ for some $\beta \in \Delta_{X}, w \in W_{X}$. Write $w=w^{\prime \prime} \circ w^{\prime}$ with $w^{\prime}\left(C_{X}\right) \subset C_{X}^{n}$ and $w^{\prime \prime} \in W_{X}^{n}$. According to (3.2), there is a sequence

$$
\left\{X_{r} \underset{\phi_{r}}{\longrightarrow} X_{r-1} \longrightarrow \cdots \underset{\phi_{1}}{\longrightarrow} X_{0}=X\right\}
$$

of modifications of type I such that $\phi_{r}^{*} \circ \cdots \circ \phi_{1}^{*} \circ w^{\prime}(\beta)=[L]+\xi\left[L^{\prime}\right]$ for some exceptional curves $L, L^{\prime}$ on $X_{r}(r \geqq 0)$, where $\xi=-1$ (resp. $\left.\xi=1\right)$ if and only if $L$ and $L^{\prime}$ lie on the same component of $X_{r}$ (resp. on the distinct component). Let $E_{r}$ be the double curve of $X_{r}$. Since $w^{\prime \prime} \circ w^{\prime}(\beta)$ $\equiv w^{\prime}(\beta) \bmod Z \cdot \Delta_{X}^{n}, \omega_{X}\left(w^{\prime}(\beta)\right)=\omega_{X}\left(w^{\prime \prime} \circ w^{\prime}(\beta)\right)=\omega_{X}(\alpha)=0$ in $J(E)$. Hence, by (2.9), (iii), $\omega_{X_{r}}\left([L]+\xi\left[L^{\prime}\right]\right)=0$ in $J\left(E_{r}\right)$. This implies $L \cap E_{r}=L^{\prime} \cap E_{r}$ by Abele's theorem. It follows that either $L$ contains $L^{\prime}$ or $L$ does not lie on the component of $X_{r}$ on which $L^{\prime}$ lies. Hence $[L]+\xi\left[L^{\prime}\right] \in R_{X_{r}}^{n}$. By definition, each $\phi_{i}$ preserves the nodal classes and so $w^{\prime}(\beta) \in R_{X}^{n}$. Consequently $\alpha=w^{\prime \prime} \circ w^{\prime}(\beta) \in R_{X}^{n}$.

\section{REFERENCES}

[1] Burns, D. and M. Rapoport, On the Torelli problem for $K 3$ surfaces, Ann. Sci. École Norm. Sup., 4 (1975), 235-274.

[2] Friedman, R. D., Hodge theory, degenerations, and the global Torelli problem, Harvard Thesis (1981).

[ 3 ] — Global smoothings of varieties with normal crossings, Ann. of Math., 118 (1983), 75-114.

[4] Friedman, R. D. and D. Morrison editors, Birational geometry of degenerations, Progress in Math. (1983), Birkhauser.

[ 5 ] Kempf, G. et al., Toroidal embeddings, I, Lecture Note in Math., vol. 339, Springer (1973).

[6 ] Kodaira, K., On compact analytic surface, II, Ann. of Math., 77 (1963), 563-626.

[ 7 ] Kulikov, V., Degenerations of $K 3$ surfaces and Enriques surfaces, Math. USSRIzv, 11 (1977), 957-989.

[ 8 ] Looijenga, E., Invariant theory for generalized root systems, Invent. Math., 61 (1980), 1-32.

[ 9 ] _ Rational surfaces with anti-canonical cycle, Ann. of Math., 114 (1981), $267-322$.

[10] Looijenga, E. and C. Peters, Torelli theorem for K3 surfaces, Compositio Math., 42 (1980), 145-186.

[11] Namikawa, Y., Type I degenerations of $K 3$ surfaces. (in Japanese), Proc. Symp. Algebraic Geometry, Tohoku University (1980). 
[12] Persson, U. and H. Pinkham, Degenerations of surfaces with trivial canonical bundle, Ann. of Math., 113 (1981), 45-66.

[13] Piatetskii-Shapiro, I. and I. Shafarevich, A Torelli theorem for algebraic surfaces of type K3, Math. USSR-Izv., 35 (1971), 530-572.

[14] Vinberg, E., Some arithmetical discrete groups in Lobatchevsky spaces, in Discrete Subgroups of Lie groups, Bombay, Oxford Univ. Press (1973), 323-348.

[15] - Discrete linear groups generated by reflections, Math. USSR-Izv., 5 (1971), (1971), 1083-1119.

Department of Mathematics

Faculty of Sciences

Nagoya University

Nagoya, 464 Japan 